\title{
Prevalência e Risco de Tabagismo entre Estudantes do Ensino Médio em Cidade do Nordeste do Brasil
}

\author{
Deborah Rose Galvão Dantas $^{a} \quad$ Adelmo de Souza Machado Neto $^{\text {b, c }}$ \\ Gilberto da Silva Matos $^{d}$ Guilherme Figueiredo da Silva $^{a}$ \\ Iurhi Henrique Guerra Pereira Pinto ${ }^{a}$ André Cavalcante Marques ${ }^{a}$ \\ Bruno de Souza Rodrigues $^{\text {a }}$ Adelmir de Souza Machado ${ }^{e, f}$ \\ a Unidade Acadêmica de Medicina, Centro de Ciências Biológicas e da Saúde, Universidade Federal de Campina Grande \\ (UFCG), Campina Grande, Brazil; b Faculdade de Tecnologia e Ciências (FTC) - Curso Medicina, Salvador, Brazil; \\ 'Escola Bahiana de Medicina e Saúde Pública - Fundação Bahiana para o Desenvolvimento da Ciência (FBDC), Salvador, \\ Brazil; ' 'Unidade Acadêmica de Estatística, Centro de Ciências e Tecnologia, Universidade Federal de Campina Grande \\ (UFCG), Campina Grande, Brazil; e Programa de Pós Graduação em Medicina e Saúde, Departamento de Medicina da \\ Faculdade de Medicina da Bahia (FMB), Universidade Federal da Bahia (UFBA), Salvador, Brazil; f'Departamento de \\ Biomorfologia, Instituto de Ciências e Saúde, Universidade Federal da Bahia, Salvador, Brazil
}

\section{Palavras Chave}

Tabagismo $\cdot$ Estudante $\cdot$ Fatores de risco $\cdot$ Estudos transversais · Brasil

\section{Resumo}

Objetivo: Descrever a prevalência e fatores de risco ao tabagismo entre estudantes de Campina Grande, Paraíba, Brasil. Método: Corte transversal com 781 estudantes de 16 escolas. Para a análise estatística utilizou-se o sistema computacional $\mathrm{R}$, teste qui-quadrado e regressão logística. Resultados: A prevalência de fumadores foi de 9,8\%; a experimentação, 31,2\%; fumadores atuais 6\%; ex-fumadores 4,6\%; expostos ao fumo ambiental, $68 \%$. Fatores de risco: conviver com fumadores, ser indiferente ao controlo da venda, considerar desnecessário o aumento da fiscalização e observar menores a comprar cigarros. Conclusão: As prevalências não apresentaram redução, contrariando a tendência observada nas últimas décadas no Brasil. Os fatores de risco estão ligados ao convívio com fumadores e à falha na prevenção e cumprimento das leis de proteção dirigidas aos adolescentes.

(C) 2017 The Author(s). Published by S. Karger AG, Basel on behalf of Escola Nacional de Saúde Pública
The Prevalence and Risk Factors of Smoking among High School Students in a City in Northeastern Brazil

\section{Keywords}

Smoking · Student · Risk factors · Cross-sectional studies . Brazil

\section{Abstract}

Objective: We aim to describe the prevalence and risk factors of smoking among students in Campina Grande, PB, Brazil. Method: A cross-sectional study was completed

\section{KARGER}

E-Mail karger@karger.com www.karger.com/pjp (c) 2017 The Author(s). Published by S. Karger AG, Basel on behalf of Escola Nacional de Saúde Pública Kareer

Open access

This article is licensed under the Creative Commons AttributionNonCommercial-NoDerivatives 4.0 International License (CC BYNonCommercial-NoDerivatives 4.0 International License (CC BY-
NC-ND) (http://www.karger.com/Services/OpenAccessLicense) NC-ND) (http://www.karger.com/Services/OpenAccessLicense) tribution of modified material requires written permission.
Deborah Rose Galvão Dantas

Unidade Acadêmica de Medicina, Centro de Ciências Biológicas e da Saúde Universidade Federal de Campina Grande (UFCG)

Hospital Universitário Alcides Carneiro, Rua Carlos Chagas, $\mathrm{S} / \mathrm{N}$

São José, CEP, Campina Grande, PB 58460-39 (Brazil)

E-Mail deborahdantas4@ hotmail.com 
with 781 students from 16 schools. We performed statistical analyses using the $R$ computing environment, $X^{2}$ test, and logistic regression. Results: The prevalence of smokers was $9.8 \%$, with $31.2 \%$ lifetime users, $6 \%$ current smokers, $4.6 \%$ former smokers, and $68 \%$ passive smokers. Risk factors included living with smokers, being indifferent to the sale control, considering an increase in surveillance to be unnecessary, and observing underage people buying cigarettes. Conclusion: The prevalence of smoking did not decrease compared to the trends observed in the recent decades in Brazil. Risk factors were linked to living with smokers and a failure to both prevent smoking and obey protection laws aimed at teenagers.

๑ 2017 The Author(s). Published by S. Karger AG, Basel on behalf of Escola Nacional de Saúde Pública

\section{Introdução}

O tabagismo representa um grave problema de saúde pública e continua a ser a principal causa prevenível de morte no mundo. Atualmente, doenças associadas ao tabagismo matam quase seis milhões de pessoas por ano, $80 \%$ delas em países em desenvolvimento $[1,2]$. De acordo com a Organização Mundial de Saúde (OMS), em 2030, oito milhões de mortes anuais serão causadas por doenças relacionadas ao tabagismo, metade das quais em indivíduos na faixa de idade produtiva [2], a menos que sejam adotadas medidas preventivas e urgentes.

No Brasil, observou-se uma redução de 35\% na percentagem de fumadores no período de 1989 a 2003 [3], o que coincide com a adoção de campanhas educativas e leis antitabagismo mais numerosas e incisivas, incluindo a proibição da propaganda através dos meios de comunicação [4]. No entanto, 178.000 mortes anuais em adultos com 35 ou mais anos foram atribuídas ao tabagismo em 2003 [5].

Posteriormente, estudos sucessivos, realizados entre 2006 e 2011 na população acima de 18 anos em 26 capitais brasileiras e no Distrito Federal, mostraram que as prevalências pouco variaram (de 16,2\% em 2006 [6] a 14,8\% em 2011 [7]), embora a percentagem dos que deixaram de fumar fosse maior a cada ano [6-9], o que deveria resultar numa redução continuada da prevalência nessa população.

A discreta variação da prevalência na população adulta nos últimos anos [8-11], mesmo com o aumento da quantidade de pessoas que pararam de fumar, pode apontar para a manutenção ou aumento da prevalência do tabagismo entre os que se iniciam, e que as medidas gover-

Prevalência e Risco de Tabagismo entre Estudantes namentais consideradas eficazes na cessação do tabagismo na população adulta podem não estar a ser tão eficientes de modo a evitar que os adolescentes comecem ou continuem a fumar.

Dados de 2010 da OMS [1] mostram que a prevalência de tabagismo está a aumentar entre os adolescentes de países em desenvolvimento, devido à publicidade ao tabaco e ao crescimento populacional nesses países. Um estudo realizado em 151 países entre 2000 e 2007 [12] concluiu que $9,5 \%$ dos estudantes entre 13 a 15 anos eram fumadores regulares. Entre os estudantes brasileiros de 12 a 16 anos avaliados de 2002 a 2005 em 12 capitais brasileiras [13], a prevalência do tabagismo variou de 6,0\% em Salvador até $23,0 \%$ em Porto Alegre. Além disso, a prevalência do tabagismo entre crianças e adolescentes brasileiros tem sido pouco relatada através de escassos estudos com metodologia diversa e dados insuficientes [14].

O Nordeste é uma das regiões mais pobres do Brasil e apresenta a segunda maior prevalência de fumadores no país, e o estado da Paraíba é o terceiro estado brasileiro onde mais se fuma, de acordo com dados recentes [15], apesar de cumprir com a maioria das recomendações da Organização Mundial de Saúde, no que respeita à proteção contra o tabagismo ambiental [16]. A cidade de Campina Grande localiza-se no estado da Paraíba, no Nordeste do Brasil, e tem uma população estimada em 389.995 habitantes, área de $594 \mathrm{~km}^{2}$; apresenta uma população e índices de desenvolvimento [17] que a podem tornar representativa de cidades de dimensão média quando comparada com outras regiões brasileiras.

$\mathrm{O}$ ensino no Brasil divide-se em três níveis: Ensino Fundamental, Ensino Médio e Ensino Superior. Os dois primeiros são ministrados em escolas públicas ou particulares, compreendendo oito anos e três anos, respetivamente. O ensino superior é oferecido em Faculdades ou Universidades. Entre os estudantes do ensino médio, concentra-se a faixa etária na qual são registados as maiores percentagens de experimentação e iniciação do tabagismo: dos 12 aos 19 anos $[1,2]$ (adolescentes), facto pelo qual esses estudantes foram escolhidos para participarem neste estudo.

O tabagismo, e as suas complicações, poderão ser reduzidos através do conhecimento da prevalência e dos fatores de risco entre adolescentes, oferecendo um cenário para uma intervenção nesse grupo populacional. Simultaneamente, estes dados poderão mostrar se as medidas governamentais para a redução do tabagismo - que se intensificaram na última década e com sucesso no que respeita à população adulta - estão a ser úteis na prevenção e redução do tabagismo entre estes adolescentes. 
Dessa forma, este trabalho tem por objetivo descrever a prevalência e os fatores de risco associados ao tabagismo entre adolescentes na cidade de Campina Grande, PB, Brasil.

\section{Método}

Foi realizado um estudo de corte transversal de agosto a dezembro de 2011.

A população foram estudantes do ensino médio da rede pública e particular de ensino (17.498 estudantes, segundo a Secretaria de Educação do Estado da Paraíba, 2009) e a amostra, de 781 (4,46\% da população).

Entre as 54 escolas de ensino médio da cidade, 32 públicas e 22 particulares (Secretaria de Educação do Estado da Paraíba, 2009), foram sorteadas 16 - sendo duas públicas e duas particulares em cada zona (norte, sul, leste e oeste) - e retiradas aleatoriamente através de sorteio simples. Foi preservada a proporção aproximada de alunos matriculados por rede pública ou particular $(70,43 \%$ e $29,57 \%$, respectivamente) nos questionários a serem aplicados, e sorteadas turmas do primeiro ao terceiro ano do ensino médio em cada escola.

Para calcular o tamanho da amostra, utilizou-se o cálculo do teste de Hipótese para uma Proporção do Laboratório de Epidemiologia do Instituto Dante Pazzanese de Cardiologia-SP, Brasil [16]. A prevalência encontrada e utilizada nesse teste (10\%) foi obtida através de estudos sobre prevalência do tabagismo entre adolescentes na cidade de João Pessoa [13] (capital do estado e com características semelhantes às de Campina Grande) há cerca de uma década, uma vez que não foram encontrados, à época, estudos na cidade onde foi realizada a pesquisa.

A prevalência esperada (7\%), e utilizada no mesmo teste, baseou-se na redução do tabagismo na população brasileira, descrita na literatura, de aproximadamente $30 \%$ da percentagem a cada 10 anos, desde 1989 até o início da década de 2000 [3]. O número da amostra foi então calculado automaticamente, encontrando-se a indicação de 554 estudantes como número mínimo a ser pesquisado, com nível de $5 \%$ de significância e poder do teste de $80 \%$.

Os critérios de inclusão foram: questionários que não fossem rasurados ou danificados, respondidos pelos estudantes que estivessem em sala de aula no momento da aplicação; estudantes que estivessem na faixa etária entre 10 a 19 anos, e que tivessem previamente assinado o Termo de Consentimento Livre e Esclarecido (TCLE) (como também os seus responsáveis, em caso de menores de 18 anos). Foram aplicados 1.000 questionários e selecionados 781 , de acordo com os critérios de inclusão. O número de estudantes entrevistados foi superior ao mínimo calculado na amostra, o que elevou o poder do teste para aproximadamente $90 \%$.

Como instrumento de avaliação, foram utilizados questionários autoaplicáveis, adaptados de Machado Neto et al. [18] (com confiabilidade), contendo dados sociodemográficos e variáveis relacionadas ao tabagismo. [19]:

Foram definidas como variáveis dependentes (OMS, 1983)

I. Fumador regular: consumidor de no mínimo, um cigarro diário por período não inferior a seis meses;

II. Fumador ocasional: consumidor de menos de um cigarro diário por período não inferior a seis meses;
III. Fumadores: fumadores regulares e ocasionais somados;

IV. Fumador atual: aquele que fez uso de algum derivado do tabaco no último mês; V. Ex-fumador: fumador regular ou ocasional que cessou há pelo menos seis meses; VI. Não-fumador: o que não se encaixa em nenhum destes conceitos;

VII. Exposto ao fumo ambiental: aquele que convive com fumadores em locais fechados, respirando o fumo lançado no ambiente;

VIII. Experimentação: utilização de pelo menos um cigarro ou um derivado do tabaco ao longo vida.

As variáveis independentes foram as seguintes: dados sociodemográficos (sexo, idade em anos, rendimento familiar, escolaridade e estado civil dos pais); consumo de tabaco e álcool pelos pais e outras pessoas da convivência do adolescente (pares, professores, familiares); tipo de tabaco utilizado; abordagem sobre tabaco, álcool e drogas pelos pais e pela escola; opinião do adolescente sobre as campanhas de atenção ao consumo de substâncias psicoativas; compra do tabaco por menores de idade e sucesso na cessação do consumo de tabaco por terceiros, observada pelo adolescente.

Para a análise estatística, utilizou-se o sistema computacional e estatístico R versão 2.15.0 [20], sendo os dados inseridos em Excel. Foi aplicada a estatística descritiva (prevalência/frequência), com significância de 0,05 ou menos $(p \leq 0,05)$. Foram utilizados o teste qui-quadrado e análise de regressão logística com o valor de $p \leq$ 0,05 e o IC $95 \%$ para neutralizar potenciais confundidores.

Os aspetos éticos foram preservados por meio da concordância da Secretaria de Ensino do Município e dos diretores das escolas nas quais o trabalho foi desenvolvido; pela aprovação do Comité de Ética em Pesquisa do Hospital Universitário Alcides Carneiro (CEP/HUAC n 20102912-055 em 15/06/2011) e pela assinatura do TCLE por cada estudante e se menor de 18 anos, também a dos seus responsáveis.

Os questionários conservaram o anonimato e foram entregues dentro de envelopes opacos lacrados, numerados de acordo com a numeração correspondente a cada TCLE e devolvidos dentro dos envelopes, que só foram abertos no momento da análise dos dados.

\section{Resultados}

A prevalência de fumadores (regulares ou ocasionais) na amostra avaliada foi de 9,8\%, sendo 2,9\% regulares, $2,8 \%$ ocasionais e $4,1 \%$ responderam serem regulares e ocasionais simultaneamente. A percentagem de fumadores exclusivamente regulares (levando-se em conta a interseção) foi de 6,9\%. A percentagem de experimentação foi de $31,3 \%$. Com relação à faixa etária, $17 \%$ iniciaram-se com menos de 12 anos, 32\% dos 12 aos 14 anos, $46 \%$ entre os 15 e 17 anos, $4 \%$ aos 19 e apenas $1 \%$ acima de 19 anos. De entre os que experimentaram, $31,2 \%$ continuaram a fumar.

Os cigarros industrializados foram os mais utilizados (90\%), com uma frequência de 1 a 5 por dia (90\%). A percentagem de fumadores atuais entre os respondentes (6\%) foi maior do que a de ex-fumadores $(3,8 \%)$. Observaram-se 531 estudantes (68\%) expostos ao fumo am-
Dantas et al. 
Table 1. Perfil dos entrevistados e frequência da abordagem sobre drogas no domicílio e na escola $(n=781)$

\begin{tabular}{|c|c|c|c|c|}
\hline \multirow[t]{2}{*}{ Variáveis } & \multicolumn{3}{|c|}{ Frequência, \% } & \multirow{2}{*}{$\begin{array}{l}p \text {-valor do teste } \\
\text { qui-quadrado }\end{array}$} \\
\hline & $\begin{array}{l}\text { todos } \\
(n=781)\end{array}$ & $\begin{array}{l}\text { não-fumadores } \\
(n=704)\end{array}$ & $\begin{array}{l}\text { fumadores } \\
(n=77)\end{array}$ & \\
\hline \multicolumn{5}{|l|}{ Sexo } \\
\hline Feminino & 59,7 & 59,7 & 59,7 & \multirow[t]{2}{*}{0,989} \\
\hline Masculino & 40,3 & 40,3 & 40,3 & \\
\hline \multicolumn{5}{|l|}{ Idade } \\
\hline 14 a 16 anos & 43,2 & 42,9 & 46,1 & \multirow[t]{6}{*}{0,301} \\
\hline 17 a 19 anos & 45,0 & 45,9 & 36,8 & \\
\hline 20 a 22 anos & 5,8 & 5,7 & 6,6 & \\
\hline 23 a 25 anos & 2,4 & 2,1 & 5,3 & \\
\hline 26 a 30 anos & 1,7 & 1,7 & 1,3 & \\
\hline Mais de 30 anos & 1,9 & 1,7 & 3,9 & \\
\hline \multicolumn{5}{|l|}{ Estado civil dos pais } \\
\hline Solteiros & 13,2 & 12,4 & 19,7 & \multirow[t]{6}{*}{0,443} \\
\hline Casados & 60,0 & 60,1 & 59,2 & \\
\hline Separados & 2,7 & 2,7 & 1,3 & \\
\hline Divorciados & 12,3 & 12,3 & 11,8 & \\
\hline Viúvos & 4,5 & 4,6 & 3,9 & \\
\hline Outro & 7,5 & 7,9 & 3,9 & \\
\hline \multicolumn{5}{|c|}{ Rendimento familiar (salários mínimos**) } \\
\hline$<1$ & 23,8 & 24,3 & 18,7 & \multirow[t]{6}{*}{0,288} \\
\hline 1 a 4 & 56,4 & 55,3 & 66,7 & \\
\hline 4 a 8 & 11,8 & 11,9 & 10,7 & \\
\hline 8 a 10 & 3,8 & 4,3 & 0,0 & \\
\hline 10 a 20 & 3,4 & 3,5 & 2,7 & \\
\hline$>20$ & 0,8 & 0,7 & 1,1 & \\
\hline \multicolumn{5}{|l|}{ Escolaridade do pai } \\
\hline Analfabeto & 11,9 & 11,8 & 13,5 & \multirow[t]{6}{*}{0,668} \\
\hline Até $1^{\circ}$ grau & 33,6 & 33,4 & 35,1 & \\
\hline Até $2^{\circ}$ grau & 23,2 & 23,1 & 24,3 & \\
\hline Até $3^{\circ}$ grau & 13,3 & 13,2 & 13,5 & \\
\hline Pós-graduado & 3,5 & 3,8 & 1,4 & \\
\hline Não sei & 14,5 & 14,7 & 12,2 & \\
\hline \multicolumn{5}{|l|}{ Escolaridade da mãe } \\
\hline Analfabeta & 9,2 & 9,2 & 9,1 & \multirow[t]{6}{*}{$0,030^{*}$} \\
\hline Até $1^{\circ}$ grau & 30,5 & 30,2 & 32,5 & \\
\hline Até $2^{\circ}$ grau & 27,1 & 26,6 & 31,2 & \\
\hline Até $3^{\circ}$ grau & 16,4 & 16,0 & 19,5 & \\
\hline Pós-graduada & 4,4 & 4,7 & 1,3 & \\
\hline Não sei & 12,4 & 13,3 & 6,4 & \\
\hline \multicolumn{5}{|c|}{ Convive com outro fumador (não os pais) } \\
\hline Sim & 49,0 & 46,7 & 70,3 & \multirow[t]{2}{*}{0,001} \\
\hline Não & 51,0 & 53,3 & 29,7 & \\
\hline \multicolumn{5}{|c|}{ Convive com outro alcoólico (não os pais) } \\
\hline Sim & 60,2 & 58,2 & 75,0 & \multirow[t]{2}{*}{0,005} \\
\hline Não & 39,8 & 41,8 & 25,0 & \\
\hline Pais conversam sobre drogas & & & & \\
\hline Nunca & 20,2 & 20,1 & 22,4 & 0,377 \\
\hline Raramente & 31,8 & 31,4 & 35,5 & \\
\hline Frequentemente & 24,0 & 24,9 & 15,8 & \\
\hline Sempre & 24,0 & 23,6 & 26,3 & \\
\hline Abordagem sobre drogas nas & & & & \\
\hline Nunca & 38,6 & 38,5 & 39,5 & 0,756 \\
\hline Uma a duas vezes por ano & 52,7 & 52,7 & 52,6 & \\
\hline 3 a 5 vezes por ano & 5,5 & 5,4 & 6,6 & \\
\hline Mais de 6 vezes por ano & 3,2 & 3,4 & 1,3 & \\
\hline
\end{tabular}


Table 2. Opinião dos entrevistados sobre questões relacionadas com o tabagismo e observação da eficácia de uma medida de contrapropaganda e controlo de venda sobre terceiros na mesma faixa etária $(n=781)$

\begin{tabular}{|c|c|c|c|c|}
\hline \multirow[t]{2}{*}{ Variáveis } & \multicolumn{3}{|c|}{ Frequência, \% } & \multirow{2}{*}{$\begin{array}{l}p \text {-valor do } \\
\text { teste qui- } \\
\text { quadrado }\end{array}$} \\
\hline & $\begin{array}{l}\text { todos } \\
(n=781)\end{array}$ & $\begin{array}{l}\text { não-tabagistas } \\
(n=704)\end{array}$ & $\begin{array}{l}\text { tabagistas } \\
(n=77)\end{array}$ & \\
\hline \multicolumn{5}{|c|}{ Deve-se aumentar o controlo sobre a venda do tabaco } \\
\hline Sim & 82,5 & 84,2 & 66,7 & \multirow[t]{3}{*}{0,001} \\
\hline Não & 12,2 & 11,1 & 22,7 & \\
\hline Indiferente & 5,3 & 4,7 & 10,6 & \\
\hline \multicolumn{5}{|c|}{ Deve-se aumentar a fiscalização sobre drogas lícitas } \\
\hline Sim & 66,8 & 69,6 & 41,6 & \multirow[t]{3}{*}{0,003} \\
\hline Não & 27,1 & 24,2 & 53,2 & \\
\hline Indiferente & 6,1 & 6,2 & 5,2 & \\
\hline \multicolumn{5}{|c|}{ Deve-se aumentar a abordagem sobre drogas nas escolas } \\
\hline Sim & 87,4 & 88,1 & 80,5 & \multirow[t]{3}{*}{0,161} \\
\hline Não & 5,7 & 5,3 & 9,1 & \\
\hline Indiferente & 6,9 & 6,6 & 10,4 & \\
\hline \multicolumn{5}{|c|}{ As campanhas antidrogas no Brasil são eficazes } \\
\hline Sim & 38,9 & 39,2 & 35,5 & \multirow[t]{3}{*}{0,810} \\
\hline Não & 50,7 & 50,4 & 53,9 & \\
\hline Indiferente & 10,4 & 10,4 & 10,6 & \\
\hline \multicolumn{5}{|c|}{ Deve-se intensificar a contrapropaganda } \\
\hline Sim & 77,9 & 78,3 & 73,7 & \multirow[t]{3}{*}{0,081} \\
\hline Não & 14,7 & 13,8 & 22,4 & \\
\hline Indiferente & 7,4 & 7,9 & 3,9 & \\
\hline \multicolumn{5}{|c|}{ Viu um menor de idade comprar cigarros } \\
\hline Sim & 71,9 & 70,2 & 87,0 & \multirow[t]{2}{*}{0,001} \\
\hline Não & 28,1 & 29,8 & 13,0 & \\
\hline \multicolumn{5}{|c|}{ Conhece qualquer campanha antitabagismo no Brasil } \\
\hline Sim & 92,3 & 92,1 & 94,8 & \multirow[t]{2}{*}{0,392} \\
\hline Não & 7,7 & 7,9 & 5,2 & \\
\hline \multicolumn{5}{|c|}{$\begin{array}{l}\text { Conhece algum adolescente que parou de fumar devido às } \\
\text { advertências nos macos de cigarros }\end{array}$} \\
\hline Sim & 8,3 & 8,2 & 9,1 & \multirow[t]{2}{*}{0,794} \\
\hline Não & 91,7 & 91,8 & 90,9 & \\
\hline
\end{tabular}

biental na sua residência (37\%), na sua escola (34\%) ou em outros ambientes (47\%).

O perfil dos entrevistados e a análise das variáveis associadas ao tabagismo estão descritos na Tabela 1 . Na Tabela 2, é apresentada a opinião dos estudantes com relação às medidas de prevenção e controlo do tabagismo no Brasil, e o facto de observarem menores de idade a comprar cigarros. As Tabelas 1 e 2 foram descritas através da análise binária (teste qui-quadrado). Os fatores estatisticamente associados ao tabagismo através da regressão logística e razões de probabilidade são descritos na Tabela 3 .

\section{Discussão}

A prevalência de fumadores regulares ou ocasionais $(9,8 \%)$ e de fumadores exclusivamente regulares $(6,9 \%)$, neste trabalho, foi semelhante às observadas na região Nordeste por Machado Neto e Cruz [21] entre estudantes de Salvador (BA) (9,6\%) e por Nascimento et al. [22], entre estudantes do Recife dos 12 aos e 20 anos (8,3\%). Bezerra et al. [23] e Carvalho et al. [24] encontraram, entre estudantes de Pernambuco dos 14 aos 19 anos, uma prevalência de 7,8 e 7,7\%, respetivamente. Pode-se notar que as prevalências não apresentaram grandes modificações no período de tempo compreendido entre 2003 e 2011.

Em outras regiões brasileiras confirma-se a semelhança ou aumento da prevalência entre os adolescentes: na
Dantas et al. 
Table 3. Coeficientes das variáveis que influenciam no tabagismo por análise de regressão logística e razões de probabilidades esti$\operatorname{madas}(n=781)$

\begin{tabular}{lllllll}
\hline Variáveis/categorias & $\begin{array}{l}\text { Coeficiente } \\
\text { estimado }\end{array}$ & Erro-padrão & $p$-valor & $\begin{array}{l}\text { Razão de } \\
\text { probabilidade }\end{array}$ & $\begin{array}{l}\text { Razão de } \\
\text { probabilidade } \\
\text { invertida }\end{array}$ & $\begin{array}{l}\text { IC (RC; 95\%) } \\
\text { (LI; LS) }\end{array}$ \\
\hline $\begin{array}{l}\text { Convive com fumador } \\
\quad \text { Sim }\end{array}$ & 0,77 & 0,2908 & 0,0082 & $\mathbf{2 , 1 6}$ & 0,46 & 1,$23 ; 3,88$ \\
$\begin{array}{l}\text { Deve-se controlar venda } \\
\quad \text { Indiferente }\end{array}$ & 1,08 & 0,5228 & 0,0382 & $\mathbf{2 , 9 6}$ & 0,34 & 1,$01 ; 8,03$ \\
$\begin{array}{l}\text { Deve-se aumentar a fiscalizacão } \\
\quad \text { Não }\end{array}$ & 1,16 & 0,2936 & 0,0001 & $\mathbf{3 , 1 8}$ & 0,31 & 1,$79 ; 5,68$ \\
$\begin{array}{l}\text { Viu um menor a comprar cigarros } \\
\quad \text { Não }\end{array}$ & $-0,01$ & 0,0120 & 0,0120 & $\mathbf{0 , 3 6}$ & $\mathbf{2 , 7 4}$ & 0,$15 ; 0,76$ \\
\hline
\end{tabular}

região Sudeste, num estudo realizado em 1975 por Stewien e Marcondes [25] na cidade de São Paulo, demonstrou $15.5 \%$ de fumadores entre os adolescentes. Num outro estudo realizado entre 2006 e 2007, realizado por Malbergier et al. [26] nas cidades de Jacareí e Diadema (SP), verificou-se uma percentagem de $13,5 \%$ de fumadores.

No Centro-Oeste, Souza e Silveira Filho [27], num estudo realizado na cidade de Cuiabá (MT) em 1998, observaram 13,6 e 11,1\% de fumadores entre adolescentes trabalhadores e não trabalhadores, respectivamente; Jesus et al. [28] num estudo realizado em 2009, na mesma cidade, observaram uma prevalência de $16 \%$, superior à observada no estudo anterior, na mesma cidade.

Prevalências semelhantes ou superiores às encontradas há décadas preocupam, pois podem indicar que, apesar dos esforços governamentais em relação ao controlo do tabagismo no Brasil e da redução observada entre adultos nos últimos anos, as medidas adotadas até agora podem não estar a ser eficazes entre os adolescentes.

A percentagem de experimentação $(31,3 \%)$ neste estudo foi elevada, o que pôde também ser observado em outros trabalhos [13, 21, 23]. A idade de iniciação foi precoce, visto que entre os inquiridos, a maior percentagem de experimentação situa-se entre os 15 e os 17 anos de idade. Todavia, uma percentagem elevada de indivíduos entre 12 e 14 anos e menores de 12 anos revela que experimentaram o tabaco. De entre os que experimentaram fumar $31,3 \%$ mantiveram o hábito, o que está de acordo com os dados da literatura, que indicam que pelo menos um terço dos que experimentam tornam-se fumadores regulares $[1,2]$.

Estes dados podem indicar o ponto-chave da adição ao tabagismo entre os jovens: a iniciação em idade muito baixa, que não está a ser solucionada através das medidas

Prevalência e Risco de Tabagismo entre Estudantes adotadas nas campanhas que visam à abstenção entre adultos. A intervenção antes da iniciação poderia não só atuar na diminuição da prevalência, como também na prevenção de doenças crónicas irreversíveis, e na morte prematura dos fumadores, que só se apresentarão décadas depois de fumado o primeiro cigarro. O tratamento do tabagismo entre adolescentes é pouco eficaz e alguns medicamentos contraindicados: a abordagem nas escolas poderia apresentar melhores resultados, principalmente as que visam orientar o adolescente a resistir às pressões sociais que o levam a fumar [29].

Neste trabalho, a prevalência de fumadores atuais foi maior do que a de ex-fumadores ( $6 \%$ vs $3,8 \%$ ), diferindo da tendência à cessação observada em relação à população adulta no Brasil em trabalhos recentes [6, 8-11], e pode sugerir que essa tendência é questionável quando se estuda a população jovem. De modo geral, os adolescentes parecem estar pouco motivados para parar de fumar. Nos países em desenvolvimento, como o Brasil, além dos problemas comuns à faixa etária, os jovens enfrentam pouca oportunidade de inserção social, desemprego, violência, desnutrição e desagregação familiar. A ampla disponibilidade, aceitação social e ausência de reações negativas a curto prazo podem também influenciar a manutenção do tabagismo [29].

A prevalência dos expostos ao fumo ambiental, encontrada neste estudo, na casa dos adolescentes (34\%), na escola (37\%) ou em outros locais frequentados por eles (47\%) persistiu elevada, quando comparada a uma estudo anterior [13], realizado no período entre 2002 e 2003. Estes dados são também preocupantes, pois apesar da redução da prevalência do tabagismo observada nas últimas décadas no Brasil, os jovens brasileiros continuam a ser expostos ao fumo do cigarro, o que pode, por si só, in- 
fluenciar na adição ao hábito e no desenvolvimento de doenças crónicas e irreversíveis $[1,2]$.

O sexo predominante entre os fumadores foi o feminino, o que se contrapõe ao reportado em estudos recentes para os fumadores adultos brasileiros, para a região Nordeste e o estado da Paraíba $[7,15]$, mas está de acordo com outros estudos entre adolescentes no Brasil e no mundo $[3,12,13,22]$, que evidenciam a gradual equiparação dos sexos entre eles. Tal facto pode ser atribuído à mudança de estratégia da propaganda do tabaco, dirigida atualmente às mulheres e crianças dos países em desenvolvimento $[1,2]$ e à maior vulnerabilidade das mulheres: apresentam maior propensão à depressão e à ansiedade, são mais impressionáveis pela publicidade do tabaco, preocupam-se mais com o controlo do peso, e nelas o metabolismo da nicotina é mais lento [30].

Mais de $80 \%$ dos inquiridos encontravam-se na faixa etária dos 14 aos 19 anos de idade: $88,2 \%$ do total, $88,8 \%$ dos não fumadores e $82,9 \%$ dos fumadores. Esta variável não apresentou diferença estatística, nem através do teste qui-quadrado, nem através da regressão logística. O rendimento familiar também não apresentou diferença estatística entre os adolescentes fumadores e não fumadores, ao contrário do observado num recente estudo multicêntrico brasileiro [15]. A escolaridade do pai e estado civil dos pais não apresentaram diferença significativa entre os adolescentes fumadores e não fumadores, tanto pelo teste qui-quadrado quanto através da regressão logística. A escolaridade da mãe apresentou diferença estatística quando se utilizou o teste qui-quadrado, mas esse resultado não se confirmou quando se utilizou a regressão logística.

Tanto o convívio com outros fumadores quanto o convívio com alcoólicos foram mais frequentes entre os fumadores, mas apenas o primeiro apresentou associação pela análise de regressão logística. Estes resultados podem estar relacionados com o exemplo e imitação, comuns na fase de instabilidade da adolescência e à pressão do grupo social do qual o adolescente faz parte $[2,28]$. A maioria dos inquiridos afirmou nunca conversar com os pais ou responsáveis sobre o tema "drogas" e afirmou que, na escola, essa abordagem se faz uma a duas vezes por ano, o que considera insuficiente.

Uma maior percentagem conhece qualquer uma das campanhas antitabagismo no Brasil, mas as considera ineficazes, acreditando que a contrapropaganda deve ser incrementada e que a publicidade do tabaco não exerce influência sobre o consumo. Mais de $90 \%$ dos respondentes desconhecem qualquer adolescente que tenha parado de fumar devido às imagens negativas veiculadas nas carteiras de cigarros. Estes resultados sinalizam que a educa- ção para a prevenção ao tabagismo em casa e na escola não tem sido desenvolvida de maneira satisfatória. Podese aqui também reforçar a ideia de que as medidas governamentais de controlo do tabagismo não têm alcançado de maneira eficiente as faixas etárias mais jovens, tanto no que respeita à contrapropaganda, quanto à publicação de advertências e imagens de pessoas doentes nas carteiras de cigarros.

A análise de regressão logística mostrou o que influenciava um adolescente a fumar tabaco: não achar necessário aumentar a fiscalização em eventos (aumento da probabilidade em 3,18 vezes), ser indiferente ao controlo da venda do tabaco (aumento da probabilidade em 2,96 vezes), e conviver com alguém que fuma (aumento da probabilidade em 2,16 vezes).

Não ver um menor a comprar cigarros exerceu uma influência negativa sobre o tabagismo. Para facilitar a compreensão desse resultado, foi invertida a razão de probabilidades, mostrando que ver um menor comprando cigarros aumentou em 2,74 vezes a probabilidade de adição e/ ou manutenção do tabagismo.

Neste estudo, mais de 70\% dos adolescentes observaram menores de 18 anos a comprar cigarros, e consideram necessária uma maior fiscalização sobre a venda e sobre o consumo do tabaco em eventos e locais frequentados por eles. A distribuição de produtos que causam dependência a menores de 18 anos é proibida no Brasil pela Lei Federal 8.069 desde 1990 [4], e a venda, pela Lei Federal 9.294 [4] desde 1996.

Apesar do facto da legislação antitabagismo ter sido incrementada na última década no Brasil [14], a venda de cigarros a menores de 18 anos continua a ser feita em larga escala. Um estudo em 30 cidades ou regiões brasileiras [31] mostrou que a maioria absoluta dos adolescentes entre 13 a 15 anos avaliados nunca foi impedida de comprar cigarros.

Assim sendo, os riscos ao tabagismo estão relacionados não apenas à pressão do grupo social em que se insere o adolescente, mas também à ausência de educação para a prevenção ao tabagismo em casa e na escola, a falhas nas medidas governamentais para o controlo do tabagismo e ao não cumprimento das leis de proteção contra o tabagismo dirigidas a essa faixa etária no Brasil.

Outros estudos fazem-se necessários para a obtenção de dados mais expressivos no que respeita ao tabagismo entre os adolescentes brasileiros.
Dantas et al. 


\section{References}

1 World Health Organization: Chapter 1: Burden: mortality, morbidity and risk factors (Internet); in: Global Status Report on NonCommunicable Diseases 2010. Geneva, WHO, 2011, chapt 1. Available from: http:// www.who.int/nmh/publications/ncd_report_chapter1.pdf.

2 World Health Organization: WHO Report on the Global Tobacco Epidemic 2011: Warning about the Dangers of Tobacco. Geneva, WHO, 2011.

3 Monteiro CA, Cavalcante TM, Moura EC, Claro RM, Szwarcwald CL: Population-based evidence of a strong decline in the prevalence of smokers in Brazil (1989-2003). Bull World Health Organ 2007;85:527-534.

4 Aliança de Controle do Tabagismo no Brasil: Legislação federal vigente sobre tabaco no Brasil (Internet). São Paulo, ACT, 2011. Available from: http://actbr.org.br/bibliote$\mathrm{ca} /$ legislacao.asp.

5 Corrêa PCRP, Barreto SM, Passos VMA: Smoking-attributable mortality and years of potential life lost in 16 Brazilian capitals, 2003: a prevalence-based study. BMC Public Health 2009;9:206.

6 Barreto SM, Passos VMA, Giatti L: Comportamento saudável entre adultos jovens no Brasil. Rev Saude Publica 2009;43(suppl 2):9-17.

7 Brasil: Ministério da Saúde, Secretaria de Vigilância em Saúde, Secretaria de Gestão Estratégica e Participativa: Vigilância de fatores de risco e proteção para doenças crônicas por inquérito telefônico: estimativas sobre frequência e distribuição sócio-demográfica de fatores de risco e proteção para doenças crônicas nas capitais dos 26 estados brasileiros e no distrito. Brasilia, Ministério da Saúde, 2012.

8 Brasil: Ministério da Saúde, Secretaria de Vigilância em Saúde, Secretaria de Gestão Estratégica e Participativa: Vigilância de fatores de risco e proteção para doenças crônicas por inquérito telefônico: estimativas sobre frequência e distribuição sócio-demográfica de fatores de risco e proteção para doenças crônicas nas capitais dos 26 estados brasileiros e no distrito (Internet). Brasília, Ministério da Saúde, 2008. Available from: http://www.ans. gov.br/images/stories/Materiais_para_pesquisa/Materiais_por_assunto/ProdEditorialANS_Vigitel_Brasil_2008_saude_suplementar.pdf.

9 Brasil: Ministério da Saúde, Secretaria de Vigilância em Saúde, Secretaria de Gestão Estratégica e Participativa: Vigilância de fatores de risco e proteção para doenças crônicas por inquérito telefônico: estimativas sobre frequência e distribuição sócio-demográfica de fatores de risco e proteção para doenças crônicas nas capitais dos 26 estados brasileiros e no distrito (Internet). Brasilia, Ministério da Saúde, 2011. Available from: http://bvsms. saude.gov.br/bvs/publicacoes/vigitel_brasil_2011_fatores_risco_doencas_cronicas. pdf.
10 Brasil: Ministério da Saúde, Secretaria de Vigilância em Saúde, Secretaria de Gestão Estratégica e Participativa: Vigilância de fatores de risco e proteção para doenças crônicas por inquérito telefônico: estimativas sobre frequência e distribuição sócio-demográfica de fatores de risco e proteção para doenças crônicas nas capitais dos 26 estados brasileiros e no distrito (Internet). Brasilia, Ministério da Saúde, 2009. Available from: http://www.abeso.org.br/uploads/downloads/75/553a24c01eb80.pdf.

11 Brasil: Ministério da Saúde, Secretaria de Vigilância em Saúde, Secretaria de Gestão Estratégica e Participativa: Vigilância de fatores de risco e proteção para doenças crônicas por inquérito telefônico: estimativas sobre frequência e distribuição sócio-demográfica de fatores de risco e proteção para doenças crônicas nas capitais dos 26 estados brasileiros e no distrito (Internet). Brasilia, Ministério da Saúde, 2010. Available from: http://bvsms. saude.gov.br/bvs/publicacoes/vigitel_2010. pdf.

12 The Global Youth Tobacco Survey Collaborative Group: Tobacco use among youth: a cross country comparison. Tob Control 2002; 11:252-270.

13 Brasil: Ministério da Saúde. Instituto Nacional do Câncer. Vigescola: vigilância de tabagismo em escolares: dados e fatos de 12 capitais brasileiras (Internet). São Paulo, Ministério da Saúde, 2004. Available from: http:// www.inca.gov.br/vigescola/docs/vigescola completo.pdf.

14 Godoy I: Prevalência de tabagismo no Brasil: medidas adicionais para o controle da doença devem ser priorizadas no Ano do Pulmão. J Bras Pneumol 2010;36:4-5.

15 Brasil: Ministério da Saúde. Instituto Brasileiro de Geografia e Estatística. Instituto Nacional do Câncer. Pesquisa nacional por amostra de domicílios: tabagismo 2008 (Internet). Rio de Janeiro, Instituto Brasileiro de Geografia e Estatística - IBGE, 2009. Available from: http://biblioteca.ibge.gov.br/visualizacao/monografias/GEBIS\%20-\%20RJ/ pnad_tabagismo_2008.pdf.

16 Paes ÂT, Okano V: Tamanho de amostra para pesquisa em ciências da saúde [Internet]. São Paulo, Laboratório de Epidemiologia e Estatística, Instituto Dante Pazzanesi de Cardiologia, 2011.

17 Federação das Indústrias do Rio de Janeiro: Índice FIRJAN de desenvolvimento (Internet). Rio de Janeiro, Federação das Indústrias do Rio de Janeiro, 2010. Available from: http://www.firjan.com.br/ifdm/consulta-aoindice/ifdm-indice-firjan-de-desenvolvimento-municipal-resultado.htm? $\mathrm{UF}=\mathrm{PB} \& \mathrm{IdC}$ idade $=250400 \&$ Indicador $=1 \& A n o=2010$.

18 Machado ADS, Andrade TM, Zacharias HP, Carvalho FM, Paula A, Machado S, et al: Reliability of a questionnaire on substance use among adolescent students, Brazil. Rev Saude Publica 2010;44:830-839.
19 World Health Organization: Smoking and Health Programme, International Union against Cancer: Guidelines for the Conduct of Tobacco-Smoking Surveys among Health Professionals : Report of a WHO Meeting held in Winnipeg, Canada, 7-9 July 1983 in Collaboration with UICC and ACS (Internet). Geneva, WHO, 1984. Available from: http://apps.who.int/iris/bitstream/10665/ 164163/1/WHA41_4_eng.pdf.

20 R Development Core Team: The R Project for Statistical Computing (Internet). Vienna, R Development Core Team, 2011. Available from: http://www.gbif.org/resource/81287.

21 Neto ASM, Cruz ÂA: Tabagismo em amostra de adolescentes escolares de Salvador-Bahia. J Pneumol 2003;29:264-272.

22 Nascimento D, Soares EA, Feitosa S, Colares $\mathrm{V}$ : O hábito do tabagismo entre adolescentes na cidade de Recife e os fatores associados. Rev Odonto Cien2004;20:348-353.

23 Costa MCO, Alves MVDQM, Santos CADST: Experimentação e uso regular de bebidas alcoólicas, cigarros e outras substâncias psicoativas/SPA na adolescência. Cien Saude Colet 2007;12:1143-1154

24 Carvalho PD de, Barros MVG de, Lima RA, Santos CM, Mélo EN: Condutas de risco à saúde e indicadores de estresse psicossocial em adolescentes estudantes do Ensino Médio. Cad Saude Publica 2011;27:2095-2105.

25 Stewien GT de M, Marcondes RS: Uso de cigarros entre adolescentes de uma escola de $1^{\circ}$ grau da cidade de São Paulo, Brasil. Rev Saude Publica 1979;13:245-253.

26 Malbergier A, Cardoso LRD, do Amaral RA: Uso de substâncias na adolescência e problemas familiares. Cad Saude Publica 2012; 28:678-688.

27 de Souza DPO, da Silveira Filho DX: Uso recente de álcool, tabaco e outras drogas entre estudantes adolescentes trabalhadores e não trabalhadores. Rev Bras Epidemiol 2007;10: 276-287.

28 Jesus FB de, Lima FCA, Martins CBDG, Matos KF de, Souza SPS de: Vulnerabilidade na adolescência: a experiência e expressão do adolescente. Rev Gaucha Enferm 2011;32:359367.

29 Moolchan ET, Ernst M, Henningfield JE: A review of tobacco smoking in adolescents: treatment implications. J Am Acad Child Adolesc Psychiatry 2000;39:682-693.

30 Perkins KA: Smoking cessation in women: special considerations. CNS Drugs 2001; 15:391-411.

31 Brasil: Ministério da Saúde, Instituto Nacional do Câncer: A situação do tabagismo no Brasil: dados dos inquéritos do Sistema Internacional de Vigilância da Organização Mundial da Saúde realizados no Brasil entre 2002 e 2009 (Internet). Rio de Janeiro, Ministério da Saúde, 2009. Available from: http://bvsms. saude.gov.br/bvs/publicacoes/inca/PDF_final_situacao_tabagismo.pdf. 